\title{
FIELD STRENGTH CORRELATOR AND AN INFRARED FIXED POINT OF THE WILSONIAN EXACT RENORMALIZATION GROUP EQUATIONS
}

\author{
Ulrich Ellwanger用 \\ Laboratoire de Physique Théorique et Hautes Energies' \\ Université de Paris XI, Centre d'Orsay, Bâtiment 210, 91405 Orsay Cedex, France
}

\begin{abstract}
The correlator of two field strengths is computed from an effective action for Yang Mills theories, which contains both gluons and an auxiliary antisymmetric tensor field for the field strength as local variables. This action allows to relate explicitly many different approaches to confinement, and is computed using Wilsonian renormalization group equations with the bare Yang Mills action as starting point. Due to the inclusion of a higher dimensional operator in the ghost sector the running gauge coupling becomes vanishingly small at a critical scale $k_{c}$, and the resulting low energy action resembles the action of a confining string theory proposed by Polyakov.
\end{abstract}

LPTHE Orsay 98-48

July 1998

\footnotetext{
*email : ellwange@qcd.th.u-psud.fr

${ }^{\dagger}$ Laboratoire associé au Centre National de la Recherche Scientifique (URA D0063)
} 


\section{Introduction}

Many different methods have been used in order to characterize the infrared behaviour of Yang Mills theories: the Wilson loop [1], the infrared behaviour of the gluon propagator [2], the monopole condensate from a dual Higgs theory [3] and corresponding analytic models [4], a confining string theory [5] and field strength correlators [6]. The area law of the Wilson loop has been related to the infrared behaviour of the gluon propagator in [7], to monopole condensates in the dual Higgs theory in [3, 4] and to field strength correlators in [6]. More recently, field strength correlators have been studied in the dual Higgs theory in [8] and in the confining string theory in [9].

In the present paper we continue the study of an effective action for Yang Mills theories, which contains both gluons and an auxiliary antisymmetric tensor field for the field strength as local variables [10]. It has the virtue of allowing for an explicit duality transformation of its abelian projection, and allows thus to relate explicitly a $1 / q^{4}$ behaviour of the gluon propagator to a monopole condensate in a dual Higgs theory. Moreover, the parameters of this effective action can be computed from the bare Yang Mills action by integrating the Wilsonian exact renormalization group equations. Note that antisymmetric tensor fields have already appeared frequently in effective descriptions of the infrared behaviour of Yang Mills theory, motivated, e.g., by confining string theories [5] and, again, duality transformations of dual Higgs theories.

The aim of the present paper is twofold: first, in chapter 2, we compute the correlator of two field strengths from the corresponding effective action. We find indeed an exponential decrease at large distances, in agreement with results on the lattice [11], from the dual Higgs model [8] and from confining strings [9]. Notably we find that the parameters which characterize the slope of the exponential decrease are not the ones which parametrize a $1 / q^{4}$ behaviour of the gluon propagator.

Second, we reconsider the Wilsonian renormalization group flow of the parameters of the Yang Mills action in the infrared regime in chapter 3. All previous approaches in this direction [12,13 have been plagued with the appearance of a Landau singularity of the running coupling constant, which did not allow the integration of the 
renormalization group equations with respect to an infrared cutoff $k^{2}$ down to $k^{2}=0$ (unless the running coupling is put to a finite value at $k^{2}=0$ by hand). By taking a higher dimensional operator in the effective action (in the ghost sector) into account, we find that the Landau singularity is avoided. Moreover, we obtain an effective action in the deep infrared regime, which corresponds to the one of an effective abelian theory and resembles the action of a confining string theory [5]. Conclusions and a discussion of the physical interpretation of this result will be given in chapter 4 .

\section{Effective Action and the Field Strength Corre- lator}

In order to define the effective action with an auxiliary field for the field strength we start with the Yang Mills partition function

$$
e^{-G(J, \chi, \bar{\chi})}=\frac{1}{N} \int \mathcal{D}(A, c, \bar{c}) e^{-S_{Y M}-S_{g}+J \cdot A+\bar{\chi} \cdot c+\chi \cdot \bar{c}}
$$

Here $S_{Y M}$ is the standard Yang Mills action,

$$
S_{Y M}=\frac{1}{4} \int d^{4} x F_{\mu \nu}^{a} F_{\mu \nu}^{a}
$$

and $S_{g}$ is the gauge fixing and ghost part:

$$
S_{g}=\int d^{4} x\left[\frac{1}{2 \alpha}\left(\partial_{\mu} A_{\mu}^{a}\right)^{2}+\partial_{\mu} \bar{c}^{a}\left(\delta_{a c} \partial_{\mu}+g f_{a b c} A_{\mu}^{b}\right) c^{c}\right]
$$

Next we multiply the right-hand side of eq. (2.1) with

$$
1=\frac{1}{N^{\prime}} \int \mathcal{D} H e^{-\frac{1}{4} \int d^{4} x\left(F_{\mu \nu}^{a}-H_{\mu \nu}^{a}\right)^{2}} .
$$

In addition we add a source $K_{\mu \nu}^{a}$ for the auxiliary field $H_{\mu \nu}^{a}$ and obtain

$$
e^{-G(J, K, \chi, \bar{\chi})}=\frac{1}{N N^{\prime}} \int \mathcal{D}(A, H, c, \bar{c}) e^{-S(A, H)-S_{g}+J \cdot A+K \cdot H+\bar{\chi} c+\chi \bar{c}}
$$

with

$$
S(A, H)=\int d^{4} x\left(\frac{1}{2}\left(F_{\mu \nu}^{a}\right)^{2}-\frac{1}{2} F_{\mu \nu}^{a} H_{\mu \nu}^{a}+\frac{1}{4}\left(H_{\mu \nu}^{a}\right)^{2}\right)
$$


Note that one could perform the Gaussian integral over $H$ in eq. (2.5) and obtain an equivalent formulation

$$
e^{-G(J, K, \chi, \bar{\chi})}=\frac{1}{N} \int \mathcal{D}(A, c, \bar{c}) e^{-S_{Y M}-S_{g}+J \cdot A+K \cdot F+K \cdot K+\bar{\chi} c+\chi \bar{c}}
$$

The effective action including the auxiliary field $H$ is defined through the Legendre transform

$$
\Gamma(A, H, c, \bar{c})=G(J, K, \chi, \bar{\chi})+J \cdot A+K \cdot H+\bar{\chi} \cdot c+\chi \cdot \bar{c} .
$$

A general effective action contains an infinite number of terms with arbitrary powers in the fields and derivatives, which are just restricted by the need to satisfy the Slavnov-Taylor identities.

Let us now consider an expansion of the $A_{\mu}^{a}$ and $H_{\mu \nu}^{a}$ dependent part of $\Gamma$ in powers of fields and derivatives:

$$
\begin{aligned}
\Gamma(A, H) & =\frac{Z}{4}\left(F_{\mu \nu}\right)^{2}-\frac{n}{2} F_{\mu \nu} H_{\mu \nu}+\frac{m^{2}}{4}\left(H_{\mu \nu}\right)^{2} \\
& +\frac{h}{2}\left(D_{\mu} \widetilde{H}_{\mu \nu}\right)^{2}+\frac{\beta}{2}\left(D_{\mu} H_{\mu \nu}\right)^{2}+\frac{1}{2 \alpha}\left(\partial_{\mu} A_{\mu}\right)^{2}+\ldots
\end{aligned}
$$

Here $\widetilde{H}_{\mu \nu}^{a}$ is defined by $\widetilde{H}_{\mu \nu}^{a}=\frac{1}{2} \varepsilon_{\mu \nu \rho \sigma} H_{\rho \sigma}^{a}$, and the covariant derivative $D_{\mu}$, acting on fields $\varphi^{a}$ in the adjoint representation of the gauge group, by

$$
D_{\mu} \varphi^{a}=\partial_{\mu} \varphi^{a}+\bar{g} f_{a b c} A_{\mu}^{b} \varphi^{c}
$$

In this approximation $\Gamma(A, H)$ thus depends on 7 parameters $Z, n, m, h, \beta, \bar{g}$ and $\alpha$, which have to be computed within some non-perturbative scheme. In the next section we will discuss the Wilsonian exact renormalization group approach, but here we proceed by discussing several important properties of $\Gamma(A, H)$.

First, the "reduced" gauge coupling $\bar{g}$ appearing in the covariant derivative (2.10) and in the non-abelian part of the field strength $F_{\mu \nu}^{a}$ has no direct physical meaning. In order to define a physical gauge coupling one first has to eliminate $H_{\mu \nu}^{a}$ from eq. (2.9) by its equations of motion. One obtains

$$
\Gamma(A)=\frac{Z_{e f f}}{4}\left(F_{\mu \nu}\right)^{2}+\ldots
$$


where the dots denote terms of higher order in the covariant derivatives which are induced by the terms $\sim h, \beta$. $Z_{\text {eff }}$ in (2.11) is given by

$$
Z_{e f f}=Z-\frac{n^{2}}{m^{2}}
$$

A physical gauge coupling (independent of field redefinitions of $A_{\mu}^{a}$ ) is now given by

$$
g_{\text {phys }}=\bar{g} / \sqrt{Z_{e f f}}
$$

Next we study the two point functions (in momentum space) of the fields $A_{\mu}^{a}$ and $H_{\mu \nu}^{a}$, as obtained from the effective action (2.9) in the Landau gauge $\alpha \rightarrow 0$. One finds

$$
\begin{aligned}
\left(\frac{\delta^{2} \Gamma}{\delta \varphi_{i}(-p) \delta \varphi_{j}(p)}\right)_{A_{\mu}^{a}, A_{\nu}^{b}}^{-1} & =\delta_{a b}\left(\delta_{\mu \nu}-\frac{p_{\mu} p_{\nu}}{p^{2}}\right) \cdot P_{A}\left(p^{2}\right) \\
P_{A}\left(p^{2}\right) & =\frac{p^{2} \beta+m^{2}}{p^{2}\left(Z m^{2}-n^{2}\right)+Z \beta p^{4}}, \\
\left(\frac{\delta^{2} \Gamma}{\delta \varphi_{i}(-p) \delta \varphi_{j}(p)}\right)_{A_{\mu}^{a}, H_{\rho \sigma}^{b}}^{-1} & =-i \delta_{a b}\left(p_{\rho} \delta_{\mu \sigma}-p_{\sigma} \delta_{\mu \rho}\right) \cdot P_{A H}\left(p^{2}\right) \\
P_{A H}\left(p^{2}\right) & =\frac{n}{p^{2}\left(Z m^{2}-n^{2}\right)+Z \beta p^{4}}, \\
\left(\frac{\delta^{2} \Gamma}{\delta \varphi_{i}(-p) \delta \varphi_{j}(p)}\right)_{H_{\rho \sigma}^{a}, H_{\kappa \lambda}^{b}}^{-1} & =\delta_{a b}\left(\delta_{\rho \kappa} \delta_{\sigma \lambda}-\delta_{\rho \lambda} \delta_{\sigma \kappa}\right) P_{H H, 1}\left(p^{2}\right) \\
+\delta_{a b}\left(\delta_{\rho \kappa} p_{\sigma} p_{\lambda}-\delta_{\rho \lambda} p_{\sigma} p_{\kappa}+\delta_{\sigma \lambda} p_{\rho} p_{\kappa}-\delta_{\sigma \kappa} p_{\rho} p_{\lambda}\right) P_{H H, 2}\left(p^{2}\right) & , \\
P_{H H, 1}\left(p^{2}\right)=\frac{1}{h p^{2}+m^{2}} \quad, & \\
P_{H H, 2}\left(p^{2}\right)=\frac{m^{2}}{\left(h p^{2}+m^{2}\right)\left(p^{2}\left(Z m^{2}-n^{2}\right)+Z \beta p^{4}\right)} & \cdot
\end{aligned}
$$

Let us first check the gluon propagator $P_{A}$ in the case where $\Gamma(A, H)$ of eq. (2.9) corresponds to the classical action $S(A, H)$ of eq. (2.6). After rescaling $H \rightarrow \Lambda H$ in eq. (2.6) in order to give it the appropriate dimension of a bosonic field (with $\Lambda$ equal to, e.g., an UV cutoff) this choice of $\Gamma(A, H)$ corresponds to the following choice of its parameters $Z, n, m, h, \beta$ and $\bar{g}$ : 


$$
\begin{aligned}
Z & =2, \\
n=m & =\Lambda, \\
h=\beta & =0 \\
\bar{g} & =g_{0} .
\end{aligned}
$$

Then one finds that $P_{A}\left(p^{2}\right)$ is given by $1 / p^{2}$ (and $Z_{\text {eff }}$ by 1 ) as it should be.

On the other hand, $P_{A}\left(p^{2}\right)$ enjoys a remarkable property if the three parameters $Z, n$ and $m$ are related such that

$$
Z_{e f f}=Z-\frac{n^{2}}{m^{2}} \rightarrow 0
$$

Then the gluon propagator $P_{A}\left(p^{2}\right)$ becomes

$$
P_{A}\left(p^{2}\right)=\frac{p^{2}+\Lambda_{c}^{2}}{Z p^{4}} \quad, \quad \Lambda_{c}^{2}=\frac{m^{2}}{\beta},
$$

i.e. for $p^{2}<<\Lambda_{c}^{2}$ the gluon propagator behaves like $1 / p^{4}$. In [10] we have emphasized that at the same time when eq. (2.16) is satisfied, the abelian projection of the action (2.9) allows for an explicit duality transformation, where the dual action corresponds to the one of an abelian Higgs model in the broken phase. The abelian projection corresponds simply to a vanishing of the reduced gauge coupling $\bar{g}$. (In this case the effective action would be invariant under a new gauge symmetry of the form $\delta A_{\mu}^{a}=\Lambda_{\mu}^{a}, \delta H_{\mu \nu}^{a}=\partial_{[\mu} A_{\nu]}^{a}$, were it not for the "gauge fixing term" $\beta / 2\left(\partial_{\mu} H_{\mu \nu}\right)^{2}$ in eq. (2.9).)

The fields appearing in the dual action are a dual abelian gauge field $B_{\mu}$, a Goldstone boson $\varphi$ and a free massless scalar $\chi$ (whose origin can be traced back to the "gauge fixing term" $\sim \beta$ ). The duality transformation is given by

$$
\begin{aligned}
\sqrt{Z} F_{\mu \nu}^{B} & =Z \widetilde{F}_{\mu \nu}-n \widetilde{H}_{\mu \nu} \\
\partial_{\nu} \varphi-n B_{\nu} / \sqrt{Z h} & =\sqrt{h} \partial_{\mu} \widetilde{H}_{\mu \nu} \\
\partial_{\nu} \chi & =\sqrt{\beta} \partial_{\mu} H_{\mu \nu}
\end{aligned}
$$

Assuming the relation (2.16), the dual action $\widetilde{\Gamma}$ becomes 


$$
\widetilde{\Gamma}(B, \varphi)=\frac{1}{4}\left(F_{\mu \nu}^{B}\right)^{2}+\frac{1}{2}\left(\partial_{\mu} \varphi-\widetilde{m} B_{\mu}\right)^{2}+\frac{1}{2}\left(\partial_{\mu} \chi\right)^{2}
$$

The mass $\widetilde{m}$ of the dual gauge field is given by

$$
\widetilde{m}^{2}=\frac{n^{2}}{Z h}=\frac{m^{2}}{h}
$$

where we assumed again (2.16) to hold.

Now we turn from previously published results to a new quantity of interest, the correlator of two field strengths $F_{\mu \nu}^{a}$. Due to the presence of a source $K_{\mu \nu}^{a}$ for the field strength in the form (2.7) for the partition function this correlator can simply be expressed in terms of the functional $G$ :

$$
\begin{aligned}
\left\langle F_{\mu \nu}^{a}(x) F_{\rho \sigma}^{b}(0)\right\rangle & =\left[-\delta_{a b}\left(\delta_{\mu \rho} \delta_{\nu \sigma}-\delta_{\mu \sigma} \delta_{\nu \rho}\right) \delta^{4}(x)+\frac{\delta G}{\delta K_{\mu \nu}^{a}(x)} \frac{\delta G}{\delta K_{\rho \sigma}^{b}(0)}\right. \\
& \left.-\frac{\delta^{2} G}{\delta K_{\mu \nu}^{a}(x) \delta K_{\rho \sigma}^{b}(0)}\right]_{K=J=0} \cdot
\end{aligned}
$$

(Here we have omitted the Schwinger strings, which we assume not to significantly affect its $x$ dependence.)

After the Legendre transform (2.8) the correlator becomes in terms of the effective action $\Gamma$

$$
\begin{aligned}
\left\langle F_{\mu \nu}^{a}(x) F_{\rho \sigma}^{b}(0)\right\rangle & =\left[-\delta_{a b}\left(\delta_{\mu \rho} \delta_{\nu \sigma}-\delta_{\mu \sigma} \delta_{\nu \rho}\right) \delta^{4}(x)+H_{\mu \nu}^{a}(x) H_{\rho \sigma}^{b}(0)\right. \\
& \left.+\left(\frac{\delta^{2} \Gamma}{\delta \varphi_{i} \delta \varphi_{j}}\right)_{H_{\mu \nu}^{a}(x) H_{\rho \sigma}^{b}(0)}^{-1}\right]_{\frac{\delta \Gamma}{\delta H_{\mu \nu}^{a}}=\frac{\delta \Gamma}{\delta A_{\mu}^{a}}=0}
\end{aligned}
$$

Assuming no vevs of the fields $H_{\mu \nu}^{a}$ and $A_{\mu}^{a}$,

$$
H_{\mu \nu}^{a}=A_{\mu}^{a}=0 \quad \text { for } \quad \frac{\delta \Gamma}{\delta H_{\mu \nu}^{a}}=\frac{\delta \Gamma}{\delta A_{\mu}^{a}}=0,
$$

the correlator is thus given by the Fourier transforms of the propagators given in eq. (2.140). Conventionally [6,8,11] the correlator is decomposed into two Lorentz invariant functions $D\left(x^{2}\right)$ and $D_{1}\left(x^{2}\right)$ : 


$$
\begin{aligned}
& \left\langle F_{\mu \nu}^{a}(x) F_{\rho \sigma}^{b}(0)\right\rangle=\left[-\delta_{a b}\left(\delta_{\mu \rho} \delta_{\nu \sigma}-\delta_{\mu \sigma} \delta_{\nu \rho}\right) D\left(x^{2}\right)\right. \\
& \left.+\frac{1}{2}\left(\frac{\partial}{\partial x_{\mu}}\left(x_{\rho} \delta_{\nu \sigma}-x_{\sigma} \delta_{\nu \rho}\right)+\frac{\partial}{\partial x_{\nu}}\left(x_{\sigma} \delta_{\mu \rho}-x_{\rho} \delta_{\mu \sigma}\right)\right) D_{1}\left(x^{2}\right)\right]
\end{aligned}
$$

After taking the rescaling of $H$ by $\Lambda$ into account, our expressions for the functions $D\left(x^{2}\right)$ and $D_{1}\left(x^{2}\right)$ become after comparing (2.24) with (2.14d):

$$
\begin{aligned}
& D\left(x^{2}\right)=\Lambda^{2} \int \frac{d^{4} p}{(2 \pi)^{4}} e^{i p x} P_{H H, 1}\left(p^{2}\right)-\delta^{4}(x) \\
& D_{1}\left(x^{2}\right)=-4 \Lambda^{2} \frac{d}{d x^{2}} \int \frac{d^{4} p}{(2 \pi)^{4}} e^{i p x} P_{H H, 2}\left(p^{2}\right)
\end{aligned}
$$

with $P_{H H, 1}$ and $P_{H H, 2}$ as in eq. (2.14d). Both functions $D\left(x^{2}\right)$ and $D_{1}\left(x^{2}\right)$ have been measured on the lattice [11], and at least $D\left(x^{2}\right)$ is well fitted by a decaying exponential

$$
D\left(x^{2}\right) \sim e^{-M|x|} \quad, \quad M \sim 1 \mathrm{GeV},
$$

for $|x| \lesssim 1 \mathrm{fm}$.

Subsequently we concentrate on the function $D\left(x^{2}\right)$, whose non-vanishing is known to be responsible for the area law of the Wilson loop, if the cluster expansion converges [6]. Note that in our case, if $\Gamma$ would correspond to the classical action $S$ and hence $h=0, m^{2}=\Lambda^{2}$, the propagator $P_{H H, 1}$ is simply $1 / \Lambda^{2}$ and thus $D\left(x^{2}\right)$ vanishes, since two $\delta$-functions cancel each other in eq. (2.25). For a general action (2.9) $D\left(x^{2}\right)$ is easily evaluated and one finds, for $|x| \neq 0$,

$$
D\left(x^{2}\right)=\frac{m \Lambda^{2}}{4 \pi^{2} h^{3 / 2}|x|} K_{1}\left(\frac{x m}{\sqrt{h}}\right)
$$

where $K_{1}$ is a Bessel function. Thus one obtains indeed an exponential decay (modulo powers) as in (2.26), with

$$
M=\frac{m}{\sqrt{h}} .
$$

The result (2.27) agrees with the one obtained in [8] on the basis of a dual Abelian Higgs model, and in [9] from a confining string theory. In all cases the inverse ex- 
ponential decay length $M$, eq. (2.28), coincides with the mass $\widetilde{m}$ of the dual gauge field, cf. the second equality in eq. (2.20).

Since our effective action (2.9) contains also the gluon field we can investigate, in how far the behaviour of $D\left(x^{2}\right)$ is related to the gluon propagator. Surprisingly there is no direct relation: a $1 / p^{4}$ behaviour of the gluon propagator as in eq. (2.17) depends crucially on the relation (2.16) to hold, i.e. on a relation between the parameters $Z$, $n$, and $m$. On the other hand, the result (2.27) does not at all depend on a relation like eq. (2.16). Even if this relation holds, the parameter $M$ in (2.28) is not directly related to the parameter $\Lambda_{c}$ characterizing the $1 / p^{4}$ behaviour of the gluon propagator, unless the parameters $h$ and $\beta$ in the effective action (2.9) happen to be close to each other. (Actually, the function $D_{1}\left(x^{2}\right)$ does depend on the parameters $Z, n$ and $m$. However, present lattice data [11] do not yet allow to study this function in detail.) Thus we see that the different pictures of confinement - based on the gluon propagator or on the field strength correlator - are not directly related.

Of course it can be argued that the result (2.27) is only a trivial consequence of our ansatz (2.9) for the effective action, whose parameters should eventually be computed from the Yang Mills Lagrangian. Note, however, that the action (2.9) implies the result (2.27) only if the parameters $h$ and $m^{2}$ turn out to be non-zero, finite and positive. The result of computation of the parameters of the action within the context of the Wilsonian exact renormalization group approach will be presented in the next section.

\section{An Infrared Fixed Point of Exact Renormaliza- tion Group Equations for Yang Mills Theories}

In the recent years much progress has been made in applying exact renormalization group equations [14] to gauge theories [12,13,15] 17]. Also sources coupled to composite fields can be introduced in this formalism [18 which allows to apply it to the present case.

The exact renormalization group approach requires the introduction of an "arti- 
ficial" infrared cutoff $k$ into the partition function (2.1). Then one exploits the facts that the corresponding $k$ dependent effective action $\Gamma_{k}$ becomes equal to the classical action $S$ in the limit $k \rightarrow \infty$ (up to additional terms determined by the modified Slavnov-Taylor identities [15,16]), and that an exact functional differential equation fixing the $k$ dependence of $\Gamma_{k}$ can be derived. Integrating this Wilsonian exact renormalization group equation from some large value $k=\Lambda$ down to $k=0$ provides us with the physical effective action $\Gamma_{k=0}$ in terms of the parameters of some "high energy" effective action $\Gamma_{\Lambda}$. By construction no ultraviolet invergences appear in this approach, if both $\Lambda$ and $\Gamma_{\Lambda}$ are assumed to be finite.

To be concrete, in the present case with a source $K$ coupled to the field strength $F$, one defines the functional $G_{k}(J, K, \chi, \bar{\chi})$ including the infrared cutoff $k$ by a corresponding modification of eq. (2.7):

$$
e^{-G_{k}(J, K, \chi, \bar{\chi})}=\frac{1}{N} \int \mathcal{D}(A, c, \bar{c}) e^{-S_{Y M}-S_{g}-\Delta S_{k}+J \cdot A+K \cdot F+K \cdot K+\bar{\chi} \cdot c+\chi \cdot \bar{c}}
$$

where $\Delta S_{k}$ implements the infrared cutoff for the gauge and ghost fields:

$$
\Delta S_{k}=\int \frac{d^{4} p}{(2 \pi)^{4}}\left[\frac{1}{2} A_{\mu}^{a}(-p) R_{\mu \nu}^{k}\left(p^{2}\right) A_{\nu}^{a}(p)+\bar{c}^{a}(-p) R_{g}^{k}\left(p^{2}\right) c^{a}(p)\right]
$$

The functions $R_{\mu \nu}^{k}$ and $R_{g}^{k}$ modify the gauge and ghost propagators such that modes with $p^{2}<<k^{2}$ are suppressed. Convenient choices are

$$
\begin{aligned}
& R_{\mu \nu}^{k}\left(p^{2}\right)=\left(p^{2} \delta_{\mu \nu}+\left(\frac{1}{\alpha}-1\right) p_{\mu} p_{\nu}\right) \frac{e^{-p^{2} / k^{2}}}{1-e^{-p^{2} / k^{2}}} \\
& R_{g}^{k}\left(p^{2}\right)=p^{2} \frac{e^{-p^{2} / k^{2}}}{1-e^{-p^{2} / k^{2}}}
\end{aligned}
$$

The effective action in the presence of the infrared cutoff $k$ is again defined through the Legendre transform

$$
\widetilde{\Gamma}_{k}(A, H, c, \bar{c})=G_{k}(J, K, \chi, \bar{\chi})+J \cdot A+K \cdot H+\bar{\chi} \cdot c+\chi \cdot \bar{c}
$$

Some expressions become more handsome when written in terms of $\Gamma_{k}$ which is given by $\widetilde{\Gamma}_{k}$ with the infrared cutoff term $\Delta S_{k}$ subtracted: 


$$
\Gamma_{k}(A, H, c, \bar{c})=\widetilde{\Gamma}_{k}(A, H, c, \bar{c})-\Delta S_{k}
$$

From the path integral (3.1) and the Legendre transform (3.2) it is straightforward to derive the exact renormalization group equations [12 18]

$$
\partial_{k} \Gamma_{k}=\frac{1}{2} \int \frac{d^{4} p}{(2 \pi)^{4}} \partial_{k} R^{k}\left(p^{2}\right)_{i j}\left(\frac{\delta^{2} \widetilde{\Gamma}_{k}}{\delta \bar{\varphi}_{\ell}(-p) \delta \varphi_{m}(p)}\right)_{j i}^{-1} .
$$

Here the fields $\varphi_{i} \equiv\left(A_{\mu}^{a}, H_{\mu \nu}^{a}, c^{a}, \bar{c}^{a}\right)$ denote all possible fields appearing as arguments of $\Gamma_{k}$ or $\widetilde{\Gamma}_{k}$, and the index $i$ corresponds to the field type and Lorentz and gauge group indices. The matrix $R_{i j}^{k}$ has non-vanishing matrix elements only in the subsectors $\left(A_{\mu}^{a}, A_{\nu}^{a}\right)$ and $\left(\bar{c}^{a}, c^{a}\right)$. The inverse functional $\left(\delta^{2} \widetilde{\Gamma}_{k} / \delta \bar{\varphi}_{\ell} \delta \bar{\varphi}_{m}\right)_{j i}^{-1}$, however, has to be constructed on the complete space spanned by $\left(A_{\mu}, H_{\mu \nu}, c, \bar{c}\right)$ including the auxiliary field $H_{\mu \nu}$.

The right-hand side of eq. (3.6) corresponds to a one loop diagram with an arbitrary number of vertices or external lines, and an insertion of $\partial_{k} R_{\mu \nu}^{k}$ or $\partial_{k} R_{g}^{k}$ into a gauge field or ghost propagator. The vertices and propagators have to be derived from the $k$ dependent effective action $\widetilde{\Gamma}_{k}$. For a given parametrization of $\Gamma_{k}$ (or $\widetilde{\Gamma}_{k}$ ), non-linear differential equations for the $k$ dependence of the corresponding parameters are obtained from eq. (3.6) by comparing equal powers of fields and/or derivatives acting on the fields on both sides.

Now we turn to a parametrization of the $A_{\mu}$ and $H_{\mu \nu}$ dependent part of $\Gamma_{k}$ in the form of eq. (2.9), where from now on the parameters $Z, n, m, h$ and $\beta$ depend on the infrared cutoff $k$. Differential equations describing the $k$ dependence of these parameters are obtained from eq. (3.6) by considering terms quadratic in $A_{\mu}$ or $H_{\mu \nu}$, and they have been derived in the Landau gauge in ref. [10].

On the right-hand side of these differential equations appears the "reduced" gauge coupling $\bar{g}$, since all vertices (either from the non-abelian part of $F_{\mu \nu}^{a}$ or from the covariant derivatives $D_{\mu}$ ) are proportional to $\bar{g}$. Thus the renormalization group equation describing the $k$ dependence of $\bar{g}$ is also needed.

In principle this renormalization group equation could be obtained from the three gluon vertex or the terms trilinear in $A_{\mu}$ in eq. (3.6), but due to the large number of contributing diagrams it is much more convenient to introduce the ghost sector and 
to consider the relations implied by the Slavnov-Taylor identities.

The simplest non-trivial parametrization of the ghost sector of $\Gamma_{k}$ is given by

$$
\begin{aligned}
\Gamma_{k}^{g h o s t} & =Z_{g} \partial_{\mu} \bar{c} D_{\mu} c \\
& \equiv Z_{g} \partial_{\mu} \bar{c}^{a} \partial_{\mu} c^{a}+Z_{g} \bar{g} f_{a b c} \partial_{\mu} \bar{c}^{a} A_{\mu}^{b} c^{c}
\end{aligned}
$$

Note that the Slavnov-Taylor identities imply that the reduced coupling $\bar{g}$ in eq. (3.7) equals the coupling $\bar{g}$ implicit in eq. (2.9). (Here we neglect modifications of the Slavnov-Taylor identities for $k \neq 0$ [15, 16] which vanish for $k \rightarrow 0$ ). Furthermore the ghost-gluon coupling receives no quantum corrections in the Landau gauge. In terms of the exact renormalization group equations this implies $\partial_{k}\left(Z_{g} \bar{g}\right)=0$ or

$$
\bar{g}^{-1} \partial_{k} \bar{g}=-Z_{g}^{-1} \partial_{k} Z_{g}
$$

Thus the $k$ dependence of $\bar{g}$ can be obtained from the $k$ dependence of the ghost wave function normalization $Z_{g}$, which is much easier to compute. Within the present parametrization of $\Gamma_{k}$ the corresponding exact renormalization group equation has also been given in [10, hence a closed set of differential equations describing the $k$ dependence of the 6 parameters $Z, n, m, h, \beta$ and $\bar{g}$ has been obtained. They have been integrated numerically, with boundary conditions such that at $k=\Lambda$ $\Gamma_{k}(A, H, c, \bar{c})$ corresponds to the classical action (cf. eq. (2.15)):

$$
\begin{array}{rlrl}
Z(\Lambda) & =2, & & n(\Lambda)=m(\Lambda)=\Lambda, \\
h(\Lambda)=\beta(\Lambda) & =0, & & \bar{g}(\Lambda)=g_{0}, \\
Z_{g}(\Lambda) & =1 . &
\end{array}
$$

As a result of the renormalization group flow we found indeed that for small $k Z_{\text {eff }}(k)$ (defined in terms of $Z, n$ and $m$ in eqs. (2.12) and (2.16)) vanishes. However, it was not possible to reach $k=0$, since a Landau singularity appeared in $\bar{g}(k)$ at some small, but finite value of $k=k_{c}$ : within the present approximation of $\Gamma_{k}$ the righthand side of the equation for $\partial_{k} \bar{g}(k)$ is negative definite and proportional to $\bar{g}^{2}$ (as in the case of one loop $\beta$ function for Yang Mills theories), thus a Landau singularity 
cannot be avoided. Clearly, this is an artifact of the neglect of the contributions of higher dimensional operators to the right-hand side of the exact renormalization group equations.

Let us now go beyond the previous simple truncation of $\Gamma_{k}$ and consider the effects of such higher dimensional operators. Since the running of $\bar{g}(k)$ is governed by the running of $Z_{g}(k)$, cf. eq. (3.8), we have to consider such contributions to the righthand side of the equation for $\partial_{k} Z_{g}(k)$. First, operators with the same powers of fields as in eq. (3.7), but involving higher derivatives, will not solve the problem: these have already been considered in the second of refs. [13], and they do not modify the negative definiteness of $\partial_{k} \bar{g}(k)$ (or the positive definiteness of $\partial_{k} Z_{g}(k)$, implying $Z_{g}\left(k_{c}\right)=0$ with $k_{c}$ finite).

Thus we proceed by adding an operator of higher powers in the fields to the ghost sector of $\Gamma_{k}$, which will contribute to the running of $Z_{g}(k)$. We replace $\Gamma_{k}^{\text {ghost }}$ of eq. (3.7) by

$$
\begin{aligned}
\Gamma_{k}^{\text {ghost }} & =Z_{g} \partial_{\mu} \bar{c} D_{\mu} c+\lambda \partial_{\mu} \bar{c} D_{\mu}\left(c F_{\nu \rho} F_{\nu \rho}\right) \\
& \equiv Z_{g} \partial_{\mu} \bar{c}^{a} \partial_{\mu} c^{a}+Z_{g} \bar{g} f_{a b c} \partial_{\mu} \bar{c}^{a} A_{\mu}^{b} c^{c} \\
& +\lambda \partial_{\mu} \bar{c}^{a} \partial_{\mu}\left(c^{a} F_{\nu \rho}^{d} F_{\nu \rho}^{d}\right)+\lambda \bar{g} f_{a b c} \partial_{\mu} \bar{c}^{a} A_{\mu}^{b} c^{c} F_{\nu \rho}^{d} F_{\nu \rho}^{d} .
\end{aligned}
$$

The action of the covariant derivative $D_{\mu}$ in the term $\sim \lambda$ in eq. (3.10) has been chosen such that the Slavnov-Taylor identities are still satisfied in a simple way: technically speaking, since the variation of $\Gamma_{k}^{\text {ghost }}$ with respect to $\partial_{\mu} \bar{c}^{a}$ is still proportional to a total covariant derivative, the effective BRST variation of the gluon field $A_{\mu}^{a}$ is still a total covariant derivative as before.

In principle, also a term quartic in the ghost fields could have been added to $\Gamma_{k}^{g h o s t}$. However, the corresponding contribution to $\partial_{k} Z_{g}$ involves only a diagram with an internal ghost propagator. This contribution is relatively small compared to the contribution obtained below in the limit where the gluon propagator becomes infrared singular, cf. our results below.

After inserting $\Gamma_{k}^{g h o s t}$ of eq. (3.10) into the exact renormalization group equation (3.6) and expanding the right-hand side to the order $\partial_{\mu} \bar{c} \partial_{\mu} c$ one obtains the contributions to $\partial_{k} Z_{g}$ which are shown diagrammatically in fig. 1. The "tadpole" diagram 
involving a ghost-ghost-gluon-gluon vertex is proportional to the coupling $\lambda$ in eq. (3.10).

A priori a huge number of diagrams contribute to the running of $\partial_{k} \lambda$, alone 15 to the order $\lambda^{0}$. Since we are not (yet) interested in precise quantitative results, but rather in the essential features of the system of differential equations, we will only take the leading contributions into account: to the orders $\lambda^{1}$ and $\lambda^{2}$ (no higher orders in $\lambda$ exist) we consider only those diagrams, which are leading in the case of an infrared singular gluon propagator, i.e. for $Z_{e f f} \rightarrow 0$.

Instead of including all the contributions to $\partial_{k} \lambda$ to the order $\lambda^{0}$, we will imitate these contributions by a small, but non-vanishing value of $\lambda$ at the starting point $k=\Lambda$. This procedure is justified, since one finds that for $k \rightarrow 0 \lambda$ becomes extremely large, and all contributions $\sim \lambda^{0}$ to $\partial_{k} \lambda$ become relatively negligeable. Also we have verified that the numerical results for $k \rightarrow 0$ are practically independent of the starting point value of $\lambda$ provided it is small enough. This is just a manifestation of universality of the Wilsonian exact renormalization group flow, i.e. generally the results for $k \rightarrow 0$ depend only very weakly on the irrelevant couplings in $\Gamma_{k}$ at the starting point $k=\Lambda$. Hence the diagrams which contribute to $\partial_{k} \lambda$ are finally just those shown in fig. 2, which are of the orders $\lambda^{1}$ and $\lambda^{2}$.

In order to write down the resulting exact renormalization group equations for $\partial_{k} Z_{g}$ and $\partial_{k} \lambda$ we use the following notations: we need the gluon propagator function $P_{A}\left(p^{2}\right)$ of eq. (2.14a), and in addition the ghost propagator $P_{G}\left(p^{2}\right)$ given by

$$
P_{G}\left(p^{2}\right)=\frac{1}{Z_{g} p^{2}}
$$

The presence of the infrared cutoff requires the replacement of $Z$ by $Z+\widetilde{R}$ in eq. (2.14a), and of $Z_{g}$ by $Z_{g}+\widetilde{R}$ in eq. (3.11), with

$$
\widetilde{R}=\frac{e^{-p^{2} / k^{2}}}{1-e^{-p^{2} / k^{2}}}
$$

for the choices in eq. (3.3). $g_{0}$ denotes the bare gauge coupling (appearing at the ghost gluon vertex), and, following the discussions below eq. (3.7) the coupling $\bar{g}$ equals $g_{0} / Z_{g}$. For a $\mathrm{SU}(\mathrm{N})$ gauge group we then obtain from figs. 1 and 2 : 


$$
\begin{gathered}
\partial_{k} Z_{g}=\int \frac{q^{2} d q^{2}}{16 \pi^{2}} \partial_{k} \widetilde{R}\left(q^{2}\right)\left[\frac{3 N g_{0}^{2}}{4} q^{2}\left(P_{A}^{2} P_{G}+P_{A} P_{G}^{2}\right)-12 N \lambda q^{4} P_{A}^{2}\right], \\
\partial_{k} \lambda=\int \frac{q^{2} d q^{2}}{16 \pi^{2}} \partial_{k} \widetilde{R}\left(q^{2}\right)\left[-\frac{9 N \bar{g}^{2}}{2} \lambda Z^{2} q^{4} P_{A}^{4}+4 \lambda^{2} q^{6} P_{A}^{2} P_{G}\right] .
\end{gathered}
$$

The presence of the term $\sim \lambda$ in $\Gamma_{k}^{g h o s t}$ in eq. (3.10) affects also the exact renormalization group equation for $\partial_{k} Z$, but not the equations of the remaining parameters $n, m, h$ and $\beta$. For completeness we present these five equations below. Their righthand sides receive contributions from the vertices from the non-abelian parts of $F_{\mu \nu}^{a}$, i.e. from the first two terms in $\Gamma(A, H)$ of eq. (2.9), and from the vertices due to the non-abelian parts of the covariant derivatives acting on $\widetilde{H}_{\mu \nu}^{a}$ or $H_{\mu \nu}^{a}$, which are thus proportional to $h$ or $\beta$. (These latter contributions are not very important numerically.)

Below $P_{A}, P_{A H}$ etc. denote the propagator functions of eqs. (2.14), with the replacements of $Z$ by $Z+\widetilde{R}$ as below eq. (3.11). Because of the required development in powers of derivatives (or external momenta) the quantities $p^{2} d P_{A} / d p^{2}$ etc. appear frequently, and we define for convenience

$$
P^{\prime} \equiv p^{2} \frac{d P}{d p^{2}} \quad, \quad P^{\prime \prime}=p^{4} \frac{d^{2} P}{\left(d p^{2}\right)^{2}}
$$

for all propagator functions $P_{A}, P_{A H}$ etc. With $\partial_{k} \equiv k^{2} d / d k^{2}$ and for a $\mathrm{SU}(\mathrm{N})$ gauge group the five exact renormalization group equations are

$$
\begin{aligned}
\partial_{k} Z= & \frac{N \bar{g}^{2}}{16 \pi^{2}} \int_{0}^{\infty} d p^{2} p^{4} \partial_{k} R^{k}\left[Z^{2}\left(\frac{31}{6} P_{A}^{3}+3 P_{A}^{2} P_{A}^{\prime}+P_{A}^{2} P_{A}^{\prime \prime}\right)\right. \\
& -n Z P_{A}\left(\frac{16}{3} P_{A} P_{A H}+\frac{8}{3} P_{A H} P_{A}^{\prime}+P_{A H} P_{A}^{\prime \prime}+\frac{10}{3} P_{A} P_{A H}^{\prime}+P_{A} P_{A H}^{\prime \prime}\right) \\
& +n^{2}\left(P_{A} P_{A H}\left(\frac{3}{2} P_{A H}^{\prime}+\frac{1}{2} P_{A H}^{\prime \prime}\right)+P_{A H}^{2}\left(\frac{5}{12} P_{A}+\frac{7}{12} P_{A}^{\prime}+\frac{1}{4} P_{A}^{\prime \prime}\right)\right. \\
& \left.+P_{A}^{2}\left(p^{-2}\left(\frac{9}{4} P_{H H, 1}^{\prime}+\frac{13}{12} P_{H H, 1}^{\prime \prime}\right)+\frac{3}{4} P_{H H, 2}+\frac{11}{12} P_{H H, 2}^{\prime}+\frac{1}{4} P_{H H, 2}^{\prime \prime}\right)\right) \\
& +Z \beta P_{A} P_{A H} p^{2}\left(8 P_{A H}+8 P_{A H}^{\prime}+2 P_{A H}^{\prime \prime}\right) \\
& +n \beta\left(P_{A H}^{2} p^{2}\left(2 P_{A H}-\frac{11}{3} P_{A H}^{\prime}-P_{A H}^{\prime \prime}\right)-P_{A} P_{A H}\left(6 P_{H H, 1}^{\prime}+\frac{8}{3} P_{H H, 1}^{\prime \prime}\right)\right.
\end{aligned}
$$




$$
\begin{aligned}
& \left.-P_{A} P_{A H} p^{2}\left(3 P_{H H, 2}+\frac{13}{3} P_{H H, 2}^{\prime}+P_{H H, 2}^{\prime \prime}\right)\right) \\
& +\beta^{2}\left(P_{A H}^{2} p^{2}\left(P_{H H, 1}+\frac{29}{6} P_{H H, 1}^{\prime}+\frac{11}{6} P_{H H, 1}^{\prime \prime}\right)\right. \\
& \left.+P_{A H}^{2} p^{4}\left(\frac{13}{3} P_{H H, 2}+5 P_{H H, 2}^{\prime}+P_{H H, 2}^{\prime \prime}\right)\right) \\
& +n h P_{A} P_{A H}\left(5 P_{H H, 1}^{\prime}+\frac{5}{3} P_{H H, 1}^{\prime \prime}\right)+h^{2} P_{A H}^{2} p^{2}\left(P_{H H, 1}+\frac{19}{6} P_{H H, 1}^{\prime}+\frac{5}{6} P_{H H, 1}^{\prime \prime}\right) \\
& \left.+\beta h P_{A H}^{2} p^{2}\left(5 P_{H H, 1}^{\prime}-\frac{5}{3} P_{H H, 1}^{\prime \prime}\right)+\frac{1}{6} Z_{g}^{2} P_{g}^{3}\right] \text {, } \\
& \partial_{k} n=\frac{N \bar{g}^{2}}{16 \pi^{2}} \int_{0}^{\infty} d p^{2} p^{4} \partial_{k} R^{k}\left[n P_{A}^{2}\left(\frac{5}{2} Z P_{A}-\frac{3}{2} n P_{A H}\right)\right. \\
& +n \beta P_{A}\left(6 p^{2} P_{A H}^{2}+3 P_{A} P_{H H, 1}+\frac{3}{2} p^{2} P_{A} P_{H H, 2}\right) \\
& -n h P_{A}^{2}\left(\frac{3}{2} P_{H H, 1}+P_{H H, 1}^{\prime}\right)-8 Z \beta p^{2} P_{A}^{2} P_{A H} \\
& -\beta^{2} P_{A H} p^{2}\left(\frac{9}{2} P_{A} P_{H H, 1}+3 p^{2} P_{A H}^{2}+3 p^{2} P_{A} P_{H H, 2}\right) \\
& -h^{2} p^{2} P_{A} P_{A H}\left(\frac{3}{2} P_{H H, 1}+P_{H H, 1}^{\prime}\right) \\
& \left.+\beta h p^{2} P_{A} P_{A H}\left(3 P_{H H, 1}+P_{H H, 1}^{\prime}\right)\right] \text {, } \\
& \partial_{k} m^{2}=\frac{N \bar{g}^{2}}{16 \pi^{2}} \int_{0}^{\infty} d p^{2} p^{4} \partial_{k} R^{k}\left[n^{2} P_{A}^{3}-4 \beta n p^{2} P_{A}^{2} P_{A H}-3(\beta+h) P_{A}^{2}\right. \\
& +\beta^{2} P_{A}\left(2 p^{4} P_{A} P_{A H}^{2}+p^{2} P_{A} P_{H H, 1}+p^{4} P_{A} P_{H H, 2}\right) \\
& \left.+h^{2} p^{2} P_{A}^{2} P_{H H, 1}\right] \text {, } \\
& \partial_{k} Z_{g}=\frac{N \bar{g}^{2}}{16 \pi^{2}} \int_{0}^{\infty} d p^{2} p^{4} \partial_{k} R^{k} Z_{g}^{2} P_{A} P_{g} \frac{3}{4}\left(P_{A}+P_{g}\right) \quad, \\
& \partial_{k} h=\frac{N \bar{g}^{2}}{16 \pi^{2}} \int_{0}^{\infty} d p^{2} p^{4} \partial_{k} R^{k}\left[n^{2} P_{A}^{2} p^{-2}\left(\frac{1}{6} P_{A}+\frac{4}{3} P_{A}^{\prime}+\frac{2}{3} P_{A}^{\prime \prime}\right)\right. \\
& +\beta n\left(-P_{A}^{2}\left(\frac{10}{3} P_{A H}+\frac{16}{3} P_{A H}^{\prime}+\frac{4}{3} P_{A H}^{\prime \prime}\right)-P_{A} P_{A H}\left(\frac{8}{3} P_{A}^{\prime}+\frac{4}{3} P_{A}^{\prime \prime}\right)\right) \\
& +h n P_{A}\left(2 P_{A} P_{A H}+2 P_{A} P^{\prime} A H-2 P_{A}^{\prime} P_{A H}\right) \\
& +\beta^{2}\left(P_{A}^{2}\left(\frac{3}{2} P_{H H, 1}+\frac{8}{3} P_{H H, 1}^{\prime}+\frac{2}{3} P_{H H, 1}^{\prime \prime}\right)+P_{A H}^{2} p^{2}\left(\frac{5}{3} P_{A}+\frac{4}{3} P_{A}^{\prime}+\frac{2}{3} P_{A}^{\prime \prime}\right)\right. \\
& \left.+P_{A} P_{A H} p^{2}\left(\frac{8}{3} P_{A H}^{\prime}+\frac{2}{3} P_{A H}^{\prime \prime}\right)+P_{A}^{2} p^{2}\left(\frac{25}{6} P_{H H, 2}+4 P_{H H, 2}^{\prime}+\frac{2}{3} P_{H H, 2}^{\prime \prime}\right)\right) \\
& +h^{2} P_{A}^{2}\left(\frac{9}{2} P_{H H, 1}+\frac{4}{3} P_{H H, 1}^{\prime}+\frac{1}{3} P_{H H, 1}^{\prime \prime}+\frac{3}{2} p^{2} P_{H H, 2}\right) \\
& +\beta h\left(-P_{A}^{2}\left(3 P_{H H, 1}+2 P_{H H, 1}^{\prime}\right)+P_{A H}^{2} p^{2}\left(P_{A}+2 P_{A}^{\prime}\right)\right. \\
& \left.\left.-P_{A}^{2} p^{2}\left(5 P_{H H, 2}+2 P_{H H, 2}^{\prime}\right)\right)\right] \text {, } \\
& \partial_{k} \beta=\frac{N \bar{g}^{2}}{16 \pi^{2}} \int_{0}^{\infty} d p^{2} p^{4} \partial_{k} R^{k}\left[n^{2} P_{A}^{2} p^{-2}\left(\frac{1}{12} P_{A}+\frac{2}{3} P_{A}^{\prime}+\frac{1}{3} P_{A}^{\prime \prime}\right)\right.
\end{aligned}
$$




$$
\begin{aligned}
& +\beta n\left(-P_{A}^{2}\left(\frac{14}{3} P_{A H}+\frac{8}{3} P_{A H}^{\prime}+\frac{2}{3} P_{A H}^{\prime \prime}\right)-P_{A} P_{A H}\left(\frac{4}{3} P_{A}^{\prime}+\frac{2}{3} P_{A}^{\prime \prime}\right)\right) \\
& +\beta^{2}\left(P_{A}^{2}\left(\frac{9}{2} P_{H H, 1}+\frac{4}{3} P_{H H, 1}^{\prime}+\frac{1}{3} P_{H H, 1}^{\prime \prime}\right)+P_{A H}^{2} p^{2}\left(\frac{16}{3} P_{A}+\frac{2}{3} P_{A}^{\prime}+\frac{1}{3} P_{A}^{\prime \prime}\right)\right. \\
& \left.+P_{A}^{2} p^{2}\left(\frac{13}{3} P_{H H, 2}+2 P_{H H, 2}^{\prime}+\frac{1}{3} P_{H H, 2}^{\prime \prime}\right)+P_{A} P_{A H} p^{2}\left(\frac{4}{3} P_{A H}^{\prime}+\frac{1}{3} P_{A H}^{\prime \prime}\right)\right) \\
& +h^{2} P_{A}^{2}\left(\frac{3}{2} P_{H H, 1}+\frac{8}{3} P_{H H, 1}^{\prime}+\frac{2}{3} P_{H H, 1}^{\prime \prime}\right) \\
& \left.-\beta h P_{A}^{2}\left(3 P_{H H, 1}+2 P_{H H, 1}^{\prime}\right)\right] .
\end{aligned}
$$

Note that all integrals are trivially ultraviolet finite since, with the present choice of $\widetilde{R}, \partial_{k} \widetilde{R}$ decreases exponentially for large $p^{2}$, and infrared finiteness is ensured by the presence of the infrared cutoff terms in the propagators. The integrals in eqs. (3.16), and hence the integration of the exact renormalization group equations for all parameters $Z, n, m, h, \beta, Z_{g}$ and $\lambda$ have to be performed numerically.

It has already been verified in [10] that near the starting point $k=\Lambda$, with the parameters as in eq. (3.9), the correct one loop $\beta$ functions are obtained. Thus both $Z_{\text {eff }}$ as defined in eq. (2.12) and $Z_{g}$ decrease for decreasing $k$, i.e. the reduced coupling $\bar{g}=g_{0} / Z_{g}$ increases. Also $\lambda$ increases for decreasing $k$, because the first term on the right-hand side of eq. (3.13) dominates.

This trend continues for some orders of magnitude of decreasing $k$, during which $Z_{\text {eff }}$ becomes extremely small. Then, at some critical scale $k_{c}$, a violent transition occurs, which is entirely governed by the two renormalization group equations (3.13) and (3.14). (It is well known that coupled non-linear differential equations can lead to quasi-singular solutions.) First, the right-hand side of eq. (3.13) turns negative, since $\lambda$ continued to increase. As a consequence $Z_{g}$ increases for decreasing $k$, i.e. the reduced coupling $\bar{g}$ decreases. This decrease is very rapid; nearly instantly $\bar{g}$ drops to a value very close to 0 . At some stage during this decrease, however, the second term on the right-hand side of eq. (3.14) starts to dominate. From this point onwards also $\lambda$ decreases for decreasing $k$.

Soon thereafter all evolution comes practically to an end, since all right-hand sides of the renormalization group equations become numerically tiny: the first terms are proportional to $1 / Z_{g}$ with $Z_{g}$ extremely large (remember that both $\bar{g}$ and $P_{G}$ behave like $1 / Z_{g}$ ), and the second terms are suppressed by a power of $\lambda k^{4}$ for $k \rightarrow 0$.

In figs. 3,4 and 5 we show our results, obtained numerically with $\bar{g}(\Lambda)=g_{0}=1.2$ 
and $\lambda(\Lambda)=0.01$, for $Z_{\text {eff }}(k), \bar{g}(k)=g_{0} / Z_{g}(k)$ and $\lambda(k)$ respectively. First, in fig. 3, we plot $\log \left(Z_{\text {eff }}\right)$ versus $t=-\log \left(k^{2} / \Lambda^{2}\right)$. The evolution is from left to right: for $k^{2}=\Lambda^{2} t$ is zero, and $t$ increases for decreasing $k$. One recognizes the decrease of $Z_{\text {eff }}$ up to $k=k_{c}$; for $k$ below $k_{c}$ (or $t$ above $t_{c}$ ) the evolution of $Z_{\text {eff }}$ stops: the right-hand side of all renormalization group equations of $Z, n, m, h$ and $\beta$ are proportional to $\bar{g}^{2}$, and for $t>t_{c} \bar{g}^{2}$ is vanishingly small.

This behaviour of $\bar{g}$ is shown in fig. 4, where we plot $\log (\bar{g})$ versus $t$. First, as described above, $\bar{g}$ increases until it becomes nearly singular. Then, at $k=k_{c}$, it drops instantly to a tiny value and remains there subsequently.

In fig. 5 we plot $\log (\lambda)$ versus $t$. One observes its steady increase, its maximum at $k=k_{c}$ and its subsequent drop to a somewhat smaller value.

We have checked the independence of our result on the initial values of $\bar{g}$ and $\lambda$ at the starting point $k=\Lambda$ : we have varied $\bar{g}(\Lambda)=g_{0}$ between 1.0 and 1.5 , and $\lambda(\Lambda)$ between 0.1 and 0.001 and obtained always qualitatively the same behaviour of $\bar{g}, \lambda$ and the other parameters, just the scale $k_{c}$ varies correspondingly.

We have not been able to describe the quasi-singular behaviour of $\bar{g}$ and $\lambda$ around the critical scale analytically. However, this behaviour becomes already apparent within a simplified toy model: it is possible to estimate the momentum integrals on the right-hand sides of eqs. (3.13) and (3.14), where the main contributions come from $q^{2} \sim k^{2} \cdot\left(-\log \left(Z_{\text {eff }}\right)\right)$. Then these equations can approximately be written in terms of $Z_{e f f}, \lambda$ and $Z_{g}$ (with $\bar{g} \sim 1 / Z_{g}, P_{G} \sim 1 / q^{2} Z_{g}$ ) as follows:

$$
\begin{aligned}
& \partial_{k} Z_{g}=\frac{c_{1}}{Z_{e f f} Z_{g}}-\frac{c_{2} \lambda k^{4}}{Z_{e f f}}, \\
& \partial_{k} \lambda=\frac{c_{3} \lambda^{2} k^{4}}{Z_{e f f} Z_{g}}-\frac{c_{4} \lambda}{Z_{e f f} Z_{g}^{2}}
\end{aligned}
$$

with $c_{1} \ldots c_{4}$ of $\mathcal{O}(1)$. Already for constant $Z_{\text {eff }}$ (note that $Z_{\text {eff }}$ varies only weakly around $k_{c}$ ) the solutions for $Z_{g}$ and $\lambda$ of the simplified system (3.17) of coupled nonlinear differential equations have the same properties as the ones shown in figs. 4 and 5. This constitutes an independent check of our previous numerical results, and shows also that the qualitative features are independent of additional small contributions from neglected diagrams, i.e. the precise values of the constants $c_{1} \ldots c_{4}$.

We can also insert the numerical values for $Z_{e f f}, Z_{g}, \lambda$ and $k$ for $k<k_{c}$ into the 
right-hand sides of the eqs. (3.17) and find that for all parameters $P=\left\{Z_{g}, \lambda\right\}$ we have

$$
\frac{k^{2}}{P} \frac{d P}{d k^{2}}<10^{-10}
$$

The same relation holds for all the other parameters $P=\{Z, n, m, h, \beta\}$ for $k<k_{c}$; this explains, why there is no visible evolution in this regime.

Strictly speaking, we have not yet obtained an analytic infrared fixed point of the exact renormalization group equations: the evolutions would vanish identically for $k \rightarrow 0$ only for $\bar{g}=0$. However, with $\bar{g} \sim 10^{-12}$ for $k<k_{c}$ we are so close to this fixed point that the tiny deviation plays no role for all practically purposes. (Still, the coupled system of exact renormalization group equations (3.13), (3.14) and (3.16) is so complicated that we did not manage to prove that analytically $\bar{g}(k) \rightarrow 0$ for $k \rightarrow 0)$.

It may be helpful to get some feeling for the critical scale $k_{c}$ where the sudden changes in the evolutions occur. In eq. (2.28) in section 2 we have introduced a scale $M=m / \sqrt{h}$, which describes the exponential decay of the correlators of two field strengths $F_{\mu \nu}$ and thus has some physical meaning. For the ratio $k_{c} / M$ we actually obtain, independently of the initial values of $\bar{g}$ and $\lambda$,

$$
k_{c} / M \sim 10^{-1}
$$

Let us now interpret our results in terms of the effective action $\Gamma(A, H)$ in the form of eq. (2.9). We have to keep in mind that this truncation constitutes both a low energy approximation (in the sense that higher derivatives or higher powers of the momenta have been neglected) and a weak field approximation, since higher powers of $F_{\mu \nu}$ and $H_{\mu \nu}$ have been omitted.

First, the fact that the reduced gauge coupling $\bar{g}$ practically vanishes turns the action into a free quadratic action of $N$ abelian gauge fields $A_{\mu}^{a}$ and $N$ antisymmetric tensor fields $H_{\mu \nu}^{a}$. This justifies a posteriori the abelian projection, which is required in order to make the duality transformation (2.18) feasible, and the omission of the Schwinger strings during the calculation of the correlator of two field strengths in eq. (2.21).

Second, we have introduced two physical dimensionful parameters in section 2: 
$\Lambda_{c}=m / \sqrt{\beta}$ characterizing the $1 / p^{4}$ behaviour of the gluon propagator, and $M=$ $m / \sqrt{h}$ characterizing the exponential decay of the correlator of two field strengths. Although the final results of the parameters $m, h$, and $\beta$ for $k<k_{c}$ depend strongly on the initial value of the gauge coupling $g_{0}$, we always find $h \sim \beta$ and hence $\Lambda_{c} \sim M$, and a dependence of $\Lambda_{c}$ and $M$ on the bare gauge coupling $g_{0}$ and the starting point $\Lambda$ (which plays implicitly the role of an ultraviolet cutoff) of the form

$$
\Lambda_{c}^{2} \sim M^{2} \sim \Lambda^{2} e^{-\left(16 \pi^{2} / 11 g_{0}^{2}\right)}\left(1+\mathcal{O}\left(g_{0}^{2}\right)\right)
$$

as it should be.

Next we consider the possible definition of a physical gauge coupling in eq. (2.13), $g_{\text {phys }}=\bar{g} / \sqrt{Z_{\text {eff }}}$ (describing, e.g., the low energy behaviour of a correlator of two static colored sources due to dressed one gluon exchange, cf. the second of refs. [13]). Both $\bar{g}$ and $Z_{\text {eff }}$ are tiny for $k<k_{c}$, but to our surprise we find that also the ratio coresponding to $g_{\text {phys }}$ is very small $\left(\sim 10^{-4}\right)$. This does not imply that Yang Mills theories are free theories, but rather that a momentum dependent gauge coupling $\alpha\left(q^{2}\right)$ tends to zero for $q^{2} \rightarrow 0$ (remember that we have neglected higher powers of derivatives in our action). Actually a similar result has been recently found on the lattice [19], and it coincides again with the descriptions of confinement based on effective abelian models as the dual Higgs model in [4], the "confining string theory" based on compact QED (with monopoles) in [5], and the gaussian approximation to field strength correlators in [6].

\section{Discussion}

In this paper we have made progress in the description of Yang Mills theories at low energies in terms of an effective action, which contains both gluons and an auxiliary field for the field strength as local fields. It has been known before that, for $Z_{\text {eff }} \rightarrow 0$, this action describes a $1 / p^{4}$ behaviour of the gluon propagator and, at the same time, its abelian projection allows for an explicit duality transformation relating it to a dual abelian Higgs model describing the condensation of monopoles.

Here we have shown that it also allows for the computation of the correlator of two field strengths; the result (2.27), however, has already been obtained before on 
the basis of the dual Higgs model [8] and the confining string theory [9].

Furthermore we have reconsidered the computation of the corresponding effective action within the Wilsonian exact renormalization group approach. We have included a higher dimensional operator in the ghost sector, which is responsible for the running of the reduced gauge coupling $\bar{g}$ via the Slavnov-Taylor identities. (The considered operator is actually the only one to this order in $F_{\mu \nu}$ consistent with the SlavnovTaylor identities, if one requires that the BRST variation of the gluon field is still a total covariant derivative.) We find that now the Landau singularity in the running gauge coupling disappears; on the contrary, the running gauge coupling becomes tiny at small scales. Clearly, the sudden jump of the running coupling at the critical scale $k_{c}$ resembles a phase transition; it is not quite clear, however, whether the same phenomenon would also appear as a function of the temperature.

Many properties of the resulting quasi-abelian effective action (always at low momenta, i.e. to lowest order in a derivative expansion) have already been discussed at the end of section 3. Let us add a final point, which is due to the smallness of $Z_{\text {eff }}=Z-n^{2} / m^{2}$ : for $Z_{\text {eff }} \rightarrow 0$ the first three terms $\sim F^{2}, F \cdot H$ and $H^{2}$ in $\Gamma(A, H)$ of eq. (2.9) become a perfect square $(Z / 4)(F-m H / \sqrt{Z})^{2}$. Then, due to the vanishing of $\bar{g}$, a field redefinition of the form $H \rightarrow H^{\prime}=H+\sqrt{Z} F / m$ makes $\Gamma\left(A, H^{\prime}\right)$ nearly independent of $F$ or the gauge fields $A$ : the term $\sim h$ involving $\partial_{\mu} \widetilde{H}_{\mu \nu}$ (for $\bar{g}=0)$ is invariant under this redefinition of $H$ due to the Bianchi identity, only in the term $\sim \beta$ expressions of the form $\partial_{\mu} F_{\mu \nu}$ appear. Apart from this term $\sim \beta$ the resulting action $\Gamma\left(H^{\prime}\right)$ has then the simple form

$$
\Gamma\left(H^{\prime}\right)=\frac{m^{2}}{4}\left(H_{\mu \nu}\right)^{2}+\frac{h}{2}\left(\partial_{\mu} \widetilde{H}_{\mu \nu}\right)^{2}
$$

which coincides with the weak field limit of the action of the universal "confining string theory" in [5], where the antisymmetric tensor field couples to the surface bounded by the Wilson loop like the field strength.

Clearly the relevance of the term $\sim \beta$ has to be better understood; leaving it aside, a coherent picture emerges: in [5] the action (4.1) is claimed to be universal in the sense that it does not depend on details (as the gauge group) of the confining gauge theory under consideration. Here we have obtained it from the integration of the Wilsonian exact renormalization group equations in the infrared regime, which 
should also be described by universality classes.

However, within the present approach it is straightforward to include higher derivative terms or momentum dependent couplings in the effective action (as, e.g., in the second of refs. [13]); this allows to recover the perturbative behaviour of the Green functions at large momenta. The approach is thus quite unique in allowing for a coherent description of the effective action both in the perturbative and nonperturbative regimes. If our results, in particular the infrared limit in the form of the action (4.1), can be confirmed by including higher dimensional operators in the effective action, a quantitative treatment of the non-perturbative regime within an expansion in powers of derivatives for a given Yang Mills theory seems to be within reach.

\section{Figure Captions}

Figure 1 : Diagrams contributing to the renormalization group flow $\partial_{k} Z_{g}$. Straight lines correspond to ghost propagators, twiggled lines to gluon propagators, and the crossed circle denotes an insertion of $\partial_{k} \widetilde{R}$ with $\widetilde{R}$ as in eq. (3.12).

Figure 2 : Diagrams contributing to the renormalization group flow $\partial_{k} \lambda$.

Figure $3: \log \left(Z_{e f f}\right)$ versus $t=-\log \left(k^{2} / \Lambda^{2}\right)$.

Figure $4: \ln (\bar{g})$ versus $t=-\log \left(k^{2} / \Lambda^{2}\right)$.

Figure $5: \ln (\lambda)$ versus $t=-\log \left(k^{2} / \Lambda^{2}\right)$ 


\section{References}

[1] K. G. Wilson, Phys. Rev. D10 (1974) 2445.

[2] S. Mandelstam, Phys. Rev. D20 (1979) 3223.

[3] A. M. Polyakov, JETP Lett. 20 (1974) 194;

G. t'Hooft, Nucl. Phys. B79 (1974) 276.

[4] M. Baker, J. S. Ball and F. Zachariasen, Phys. Rev. D37 (1988) 1036 and 3785; Phys. Rev. 209 (1991) 73;

S. Maedan and T. Suzuki, Progr. Theor. Phys. 81 (1989) 229.

[5] A. M. Polyakov, Nucl. Phys. B486 (1997) 23;

M. C. Diamantici, F. Quevedo and C. A. Trugenberger, Phys. Lett. B396 (1997) 115.

[6] H. G. Dosch, Phys. Lett. B190 (1987) 177;

H. G. Dosch and Y. Simonov, Phys. Lett. B205 (1988) 339;

Y. Simonov, Nucl. Phys. B324 (1989) 67, and hep-ph/9709344.

[7] G. West, Phys. Lett. B115 (1982) 468.

[8] M. Baker, N. Brambilla, H. G. Dosch and A. Vairo, HD-THEP-97-60, hepph/9802273.

[9] D. V. Antonov, hep-th/9707245 and hep-th/9710144.

[10] U. Ellwanger, LPTHE Orsay 97-44, hep-ph/9710326.

[11] A. Di Giacomo and H. Panagopoulos, Phys. Lett. B285 (1992) 133;

M. D'Elia, A. Di Giacomo and E. Meggiolaro, Phys. Lett. B408 (1997) 315;

A. Di Giacomo, E. Meggiolaro and H. Panagopoulos, hep-lat/9603017;

G. Bali, N. Brambilla and A. Vairo, Phys. Lett. B421 (1998) 265.

[12] M. Reuter and C. Wetterich, Nucl. Phys. B417 (1994) 181 and Phys. Rev. D56 (1997) 7893;

B. Bergerhoff and C. Wetterich, Phys. Rev. D57 (1998) 1591. 
[13] U. Ellwanger, M. Hirsch and A. Weber, Z. Phys. C69 (1996) 687 and Eur. Phys. J. C1 (1998) 563.

[14] K. G. Wilson and I. Kogut, Phys. Rep. 12 (1974) 15;

F. Wegner, in: Phase Transitions and Critical Phenomena, Vol. 6, eds. C. Domb and M. Green (Academic Press, NY 1975);

J. Polchinski, Nucl. Phys. B231 (1984) 269;

C. Wetterich, Phys. Lett. B301 (1993) 90;

T. Morris, Int. J. Mod. Phys. A9 (1994) 2411.

[15] G. Keller and C. Kopper, Phys. Lett. B273 (1991) 323;

C. Becchi, in: Elementary Particles, Field Theory and Statistical Mechanics, eds. M. Bonini, G. Marchesini and E. Onofri, Parma University 1993;

M. Bonini, M. D’Attanasio and G. Marchesini, Nucl. Phys. B418 (1994) 81, ibid. B421 (1994) 429, B437 (1995) 163, Phys. Lett. B346 (1995) 87.

[16] U. Ellwanger, Phys. Lett. B335 (1994) 364.

[17] M. D'Attanasio and T. Morris, Phys. Lett. B378 (1996) 213 ;

M. Bonini and F. Vian, Nucl. Phys. B511 (1998) 479.

[18] U. Ellwanger, C. Wetterich, Nucl. Phys. B423 (1994) 137.

[19] Ph. Boucaud et al., Lattice calculation of $\alpha_{s}$ in momentum scheme, Orsay preprint LPTHE 98/49 

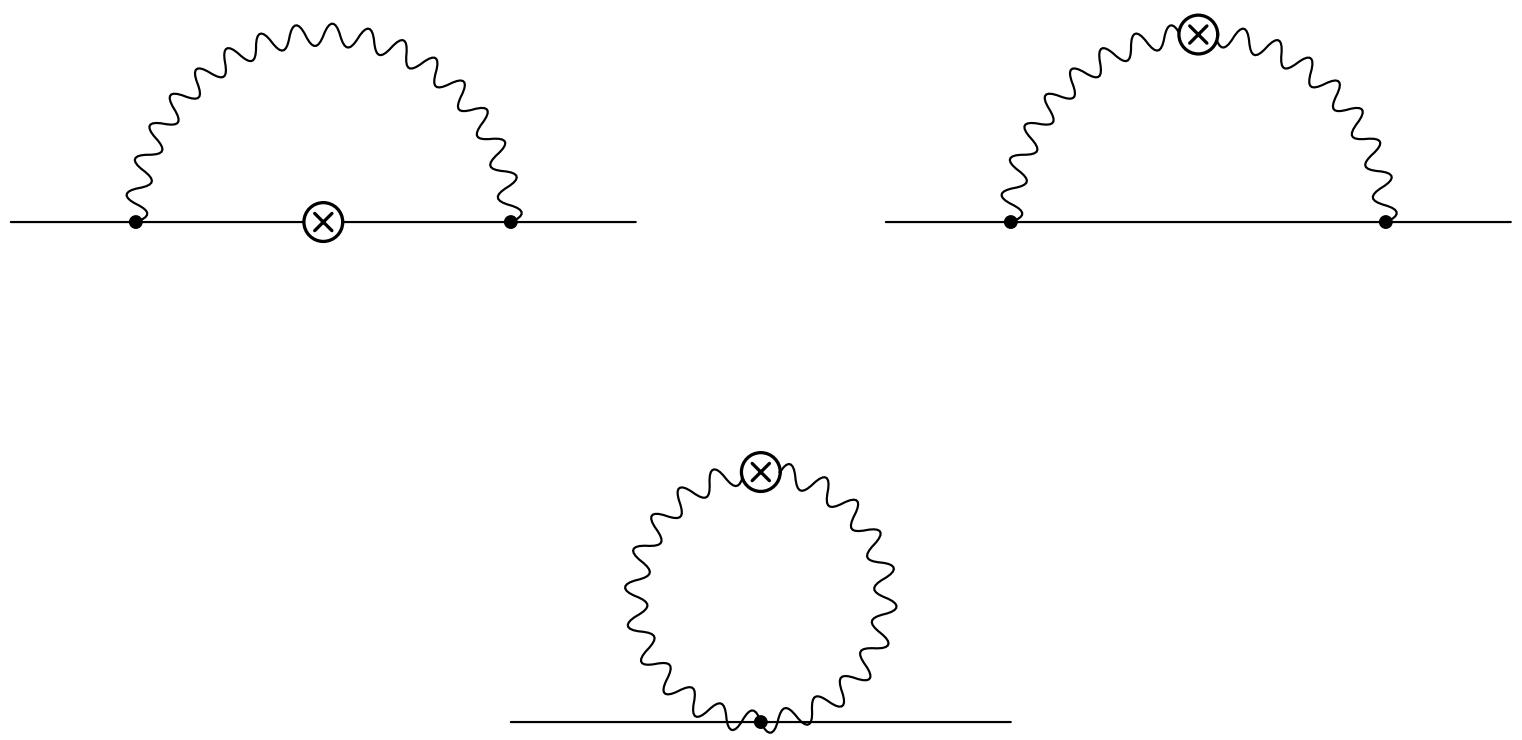

Figure 1
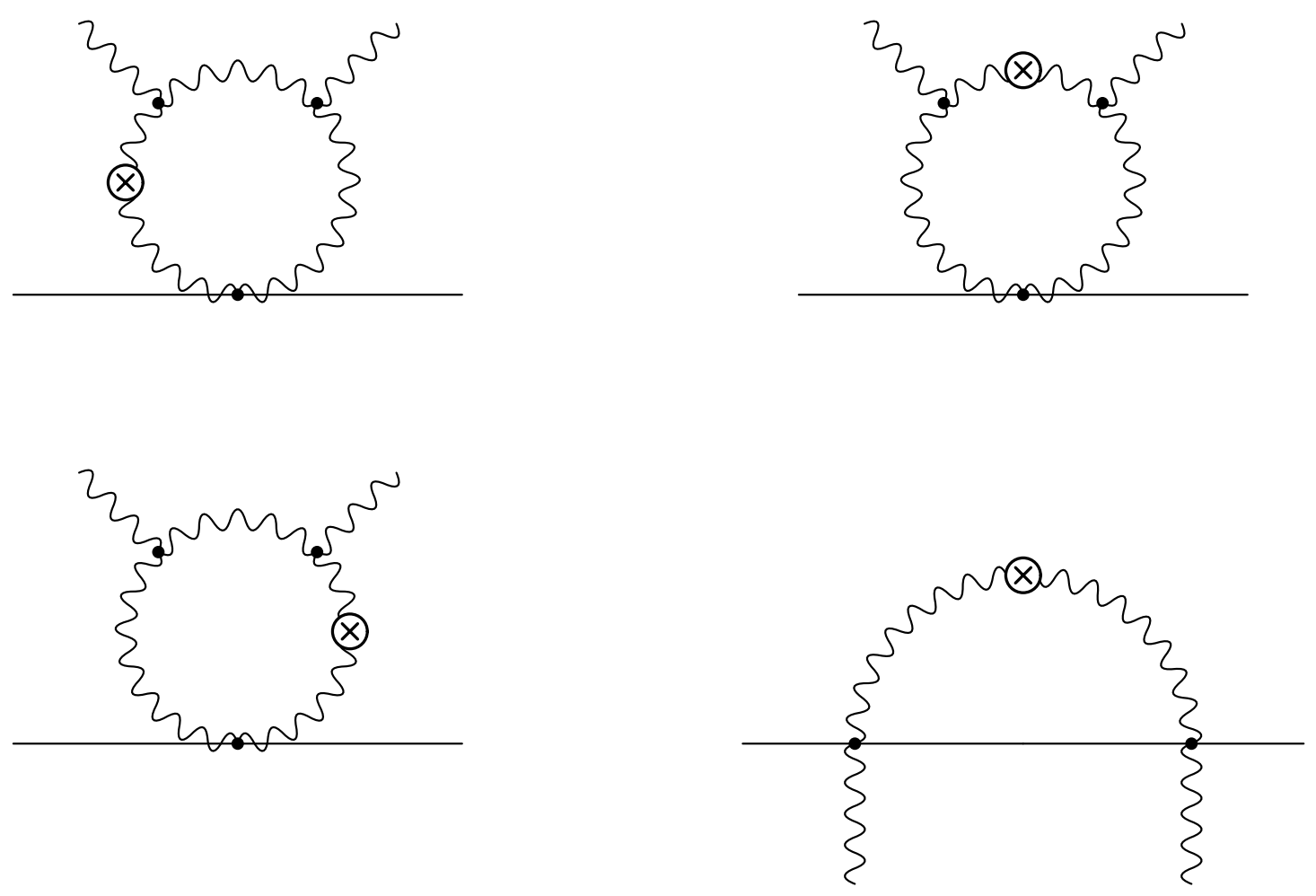

Figure 2 


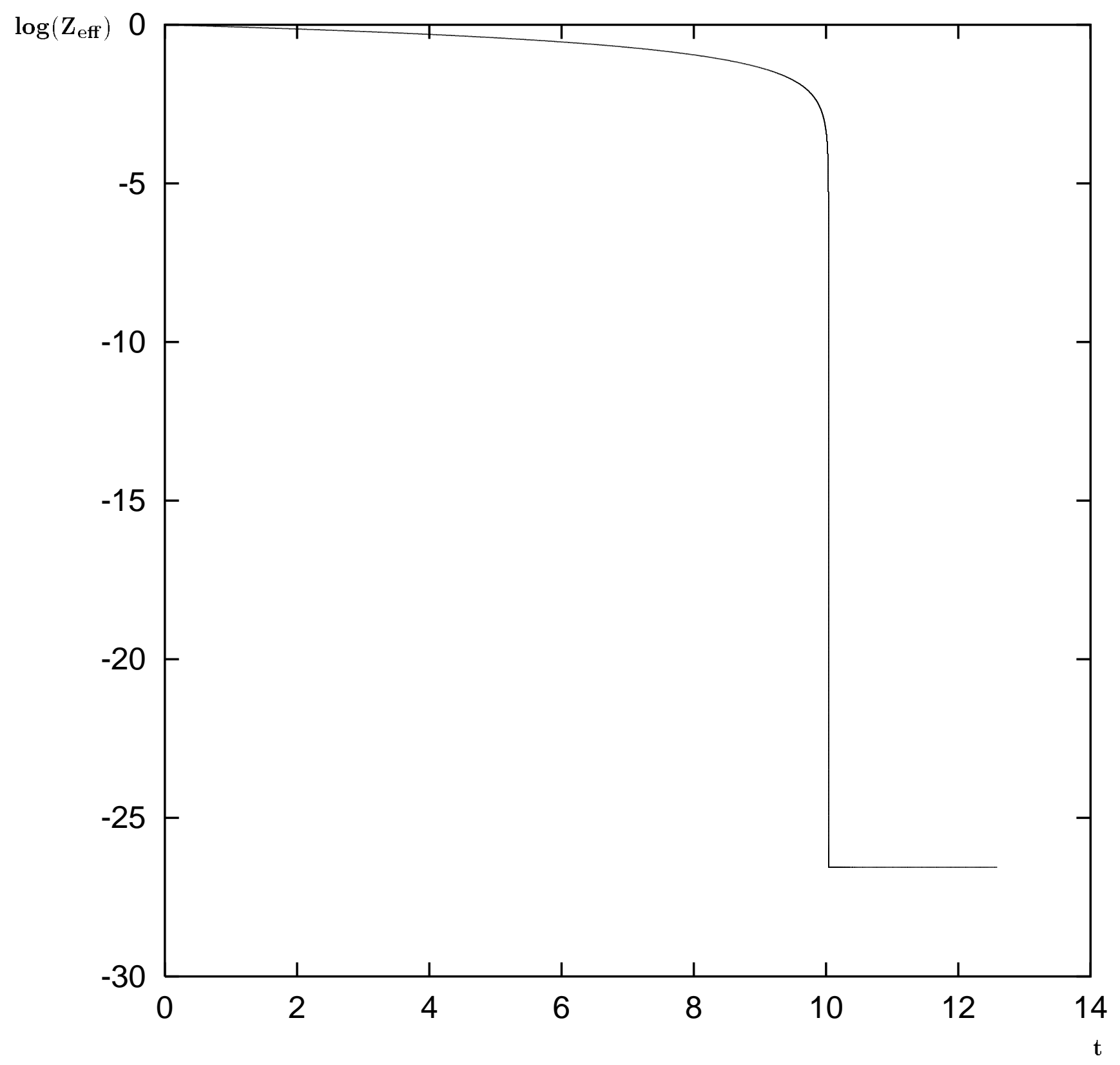

Figure 3 


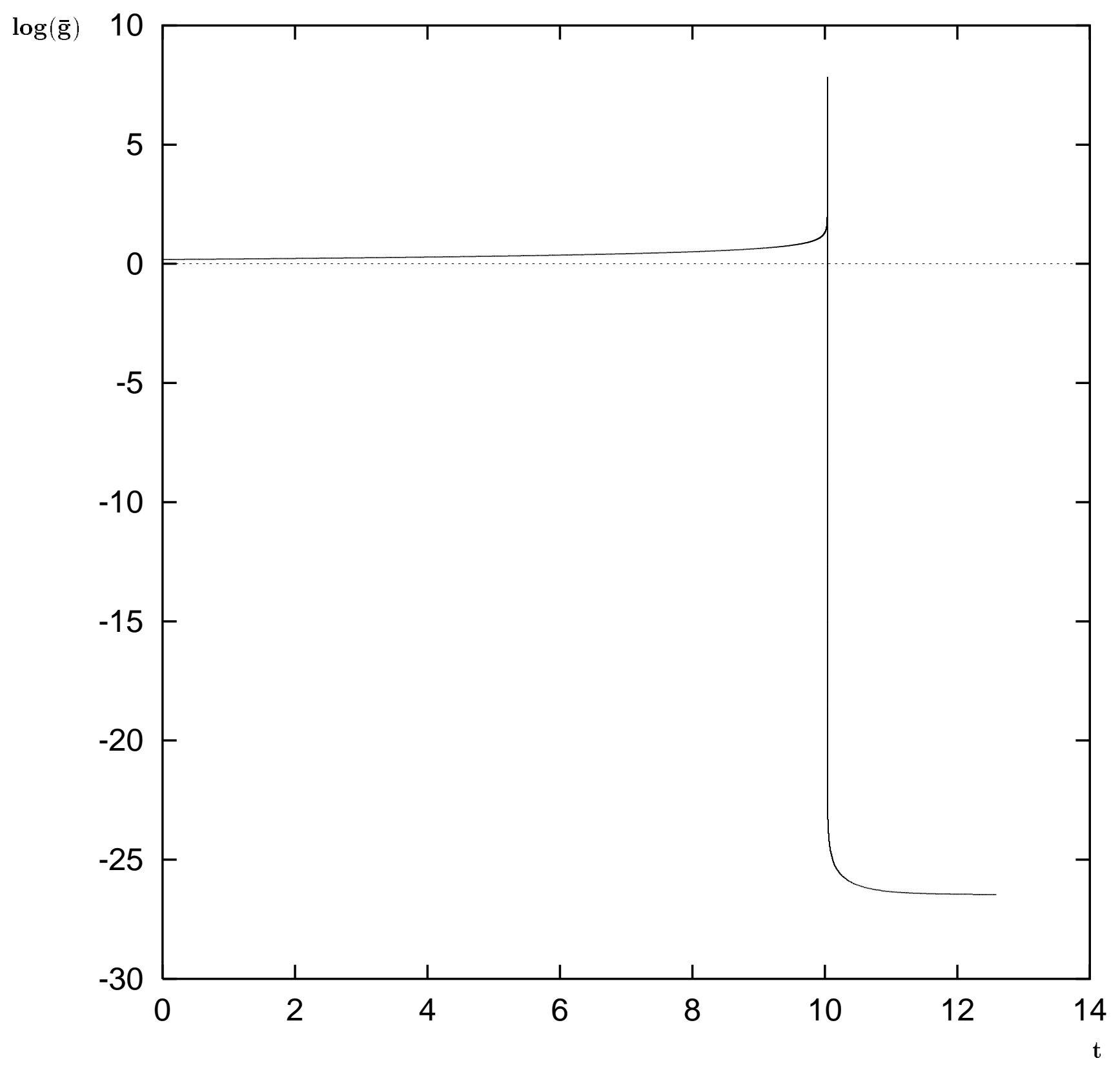

Figure 4 


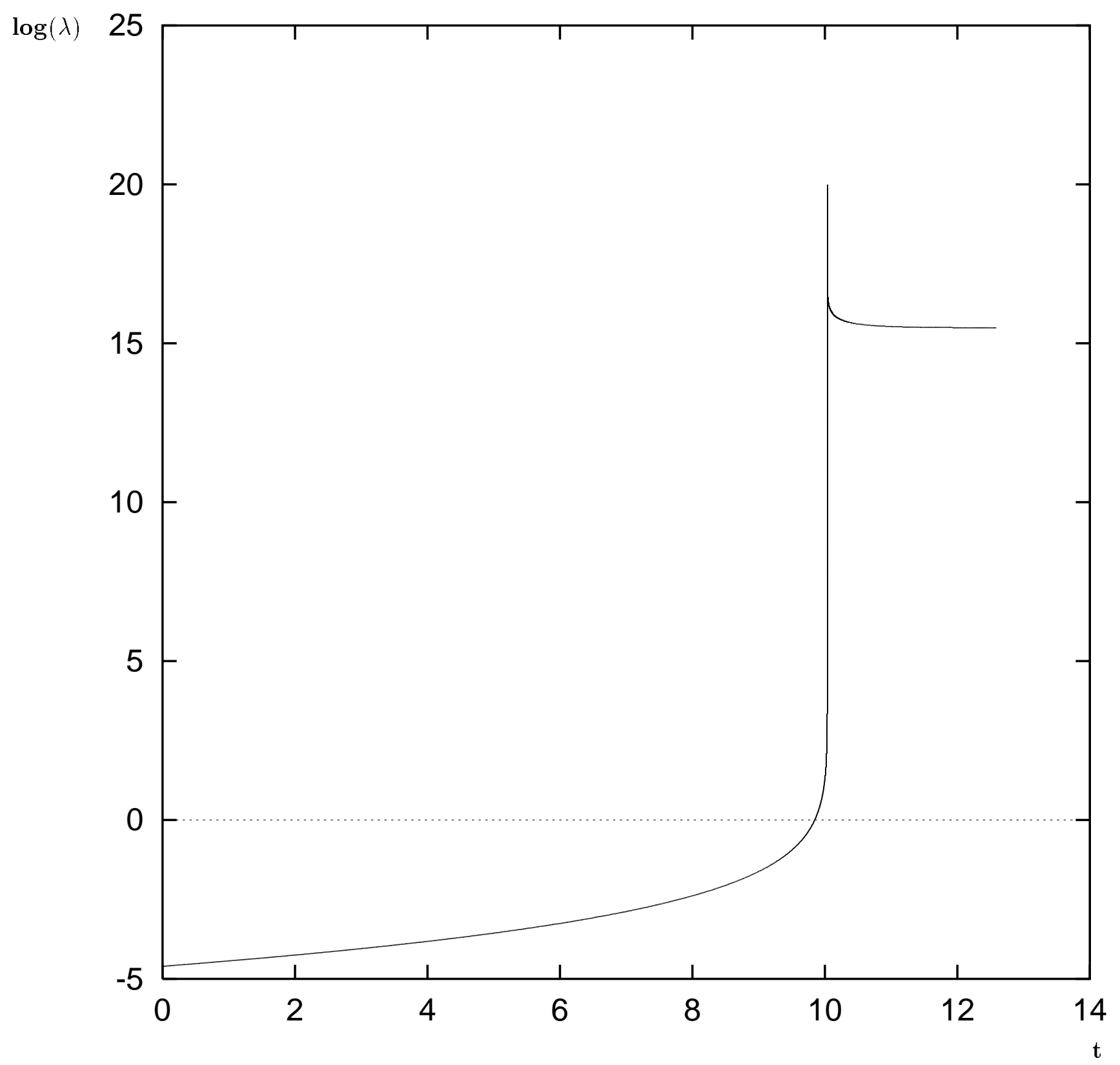

Figure 5 